\title{
Improvement of Wear Resistance in Laser Shock-Peened Copper Contacts
}

\author{
Donghyuck Jung ${ }^{1, \nabla}$, Changkyoo Park ${ }^{2, \nabla}$, Eun-Joon Chun ${ }^{3}$, and Yoon-Jun Kim ${ }^{1, *}$ \\ ${ }^{I}$ Department of Materials Science and Engineering, Inha University, Incheon 22212, Republic of Korea \\ ${ }^{2}$ Laser and Electron Beam Application Department, Korea Institute of Machinery and Materials, Daejeon 34103, Republic of Korea \\ ${ }^{3}$ Department of Nano Materials Science and Engineering, Kyungnam University, Changwon 51767, Republic of Korea
}

\begin{abstract}
This study investigated the influence of laser shock peening without coating (LSPw/oC) on the degradation of copper electrical contacts. A theoretical calculation of the plastic-affected depth (PAD) induced by LSPw/oC was performed, based on the laser-induced plasma pressure along with the Hugoniot elastic limit of our LSPw/oC experimental conditions. The theoretical PAD was obtained approximately $650 \mathrm{\mu m}$ from the surface for the LSPw/oC at the laser energy density of $5.3 \mathrm{GW} / \mathrm{cm}^{2}$. Various characterization methods such as the Vicker's hardness test, residual stress test, and electron backscattered diffraction (EBSD) mapping indicated the PAD may play a significant role in laser induced effective depth for LSPw/oC. At a laser energy density of $5.3 \mathrm{GW} / \mathrm{cm}^{2}$, the laser shock-peened copper showed approximately double the surface hardness as compared to the pure copper. This was attributed to grain refinement, which was confirmed by measuring average grain sizes, and by observing mechanical twin structures from the EBSD analysis. Additionally, a compressive residual stress was induced down to the PAD but gradually switched to a tensile residual stress below PAD. The surface hardening effect conferred by LSPw/oC to the pure copper surface resulted in excellent wear resistance, i.e., a low coefficient of friction and wear loss. As a result, the contact exhibited lower electrical resistance following the fretting friction test compared to pure copper; this would result in a significant delay in electrical contact failure.
\end{abstract}

(Received May 25, 2020; Accepted July 01, 2020)

Keywords: laser shock peening without coating, copper, wear resistance, surface hardening, residual stress, contact failure

\section{Introduction}

Electrical failure can be caused by the friction-induced wear debris of materials on the metallic contacts of switches and power connectors, which are widely used as electrical components [1,2]. In particular, electrical devices used in transportation are subjected to a variety of conditions, which can cause the malfunctioning of electrical contacts. Increased wear can occur on the electrical contacts of electrical components used in automobiles because of the small amplitude of reciprocal motion of the contacts produced by vibrations during driving [3]. This causes fretting wear, followed by the corrosion of wear debris. The agglomeration

\footnotetext{
Changkyoo Park and Donghyuck Jung contributed equally to this work. - 정동혁: 석사과정, 박창규: 연구원, 천은준·김윤준: 교수 *Corresponding Author: Yoon-Jun Kim [Tel: +82-32-860-7531, E-mail: yoonjun@inha.ac.kr] Copyright (c) The Korean Institute of Metals and Materials
}

of corroded wear debris creates an insulating layer at the electrical contacts, interrupting the transmission of electrical signals. Eventually, the fretting corrosion at the contact junction will cause an electrical failure [4-6].

Copper has been widely used to fabricate electrical contacts because of its low cost and high electrical conductivity [7]. To improve the electrical conductivity and corrosion resistance of copper, various materials, including tin, gold, and silver, have been employed as a coating layer. However, the high expense and problems with preparing such coatings hinder the usage of these materials [8-10]. Also, the effect of $\mathrm{Cu}$ grain size on fretting wear resistance has been an issue in efforts to maintain robust electrical contact [11-13].

Laser shock peening (LSP) is a mechanical surface treatment process. A specimen can be prepared either with or without a thin coating layer (e.g., black paint or $\mathrm{Al}$ and $\mathrm{Cu}$ foils), which absorbs the laser beam energy. Transparent 
materials such as glass or water, located on the specimen, are employed as a confining medium. High-energy laser pulses are applied to a thin coating layer (or directly on the specimen when no coating layer is used) through the confining medium. Then, the coating layer (or specimen) vaporizes and forms a plasma between the specimen and confining medium. By repeating the laser pulse application, a high-pressure plasma is created, resulting in the propagation of shock waves into the specimen. Plastic deformation, compressive residual stress, and grain refinement are introduced at the sub-surface of the specimen if the shock wave is sufficiently large [14]. Subsequently, the laser shock-peened specimen will exhibit superior fatigue strength, wear resistance, and corrosion resistance $[15,16]$.

To the best of our knowledge, only a few studies have investigated the influence of the LSP process on the mechanical properties and microstructures of $\mathrm{Cu}$ [17-19]. Moreover, the effect of this process on fretting corrosion has not been adequately studied. Therefore, in this study, laser shock peening without coating $(\mathrm{LSPw} / \mathrm{oC})$ process was performed on pure $\mathrm{Cu}$ metal using different laser energy densities, to investigate the impact of this process on the mechanical properties and microstructures of $\mathrm{Cu}$. As a result, increased microhardness, compressive residual stress, and grain refinement were introduced at the near-surface of the pure $\mathrm{Cu}$ specimen used in this study. A fretting test was then conducted to examine whether LSPw/oC could improve the durability of electrical contacts.

\section{Experimental details}

A high purity $\mathrm{Cu}$ plate (purity: $99.99 \mathrm{wt} \%$ ), hereafter referred to as the base metal (BM), was annealed at $180^{\circ} \mathrm{C}$ for $1 \mathrm{hr}$. A Q-switch Nd:YAG laser (Powerlite Furie, Continuum Inc.) with a wavelength of $1064 \mathrm{~nm}$, pulse duration of $12 \mathrm{~ns}$, and repetition rate of $10 \mathrm{~Hz}$ was used for the LSPwC process as shown in Figure 1 [20]. The laser beam diameter was $2 \mathrm{~mm}$ with a uniform flat-top energy distribution. Distilled water was used as the confining medium, and no ablative coating material was employed. The overlap ratio of the laser beam was $50 \%$ in both the $\mathrm{x}$ - and $\mathrm{y}$-directions, corresponding to the longitudinal and transverse directions, respectively, as sketched in Fig. 1(d) [21, 22]. The

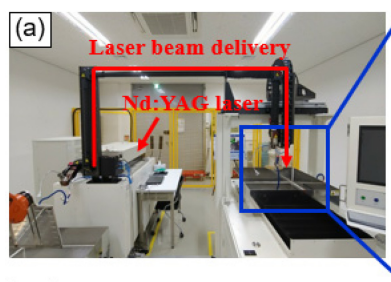

(c)

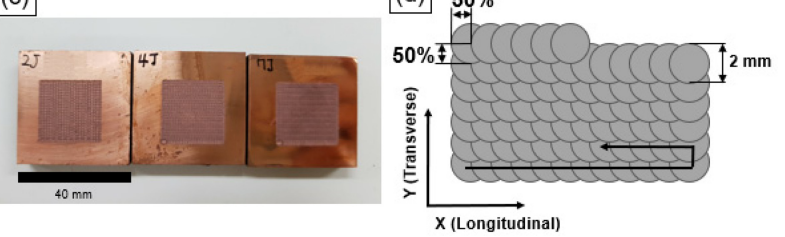

Fig. 1. Experimental set-up for the laser shock peening (LSP) without coating process of copper. (a) Q-switch Nd:YAG laser instrument, (b) Specimen holder, (c) LSP Cu specimens, and (d) schematic of laser overlaps. [20]

applied laser energies were 2,4 , and $7 \mathrm{~J}$, corresponding to laser energy densities of $5.3,10.6$, and $18.6 \mathrm{GW} / \mathrm{cm}^{2}$, respectively.

The hardness of the BM and LSP Cu were measured using Vickers hardness tester (MMT-X7, Matsuzawa) with a load of $200 \mathrm{gf}$ and holding time of $10 \mathrm{~s}$. The qualitative analysis of $\mathrm{BM}$ and LSP $\mathrm{Cu}$ was performed using an X-ray diffractometer (SmartLab, Rigaku) with the $\mathrm{Cu}-\mathrm{K} \alpha$ radiation. Additionally, the residual stress was measured at every 200 $\mu \mathrm{m}$ of depth by X-ray diffractometer (D/MAX-2500/PC, Rigaku) with $\mathrm{Cu}-\mathrm{K} \alpha$ radiation using a two-angle $\sin 2 \Psi$ technique at the $\{111\}$ plane. For electron backscatter diffraction (EBSD, Quanta 200 FEG, FEI) analysis, specimens with dimensions of $10 \mathrm{~mm} \times 10 \mathrm{~mm}$ were prepared using a low speed cutting wheel. Then, they were fine polished using 1 and $0.04 \mu \mathrm{m}$ colloidal silica suspension. The EBSD analysis was conducted using a $5.5 \mu \mathrm{m}$ beam step size. OIM AnalysisTM v8 was employed to determine the average grain size.

Wear resistance was assessed by fretting test, utilizing a reciprocating friction instrument (RFW160, NeoPlus) shown in Figure 2. The average surface roughness, $R_{a}$, of the polished specimens used for the fretting test was less than 0.2 $\mu \mathrm{m}$. Fine-polished $\mathrm{Cu}$ specimens with dimensions of $40 \mathrm{~mm}$ $(\mathrm{W}) \times 40 \mathrm{~mm}(\mathrm{D}) \times 10 \mathrm{~mm}(\mathrm{H})$ were used for the fretting test. A S45C steel ball with a $9.5 \mathrm{~mm}$ diameter was used as the counter material. The fretting test was conducted for 10,000 fretting cycles at a normal load of $10 \mathrm{~N}$, frequency of 


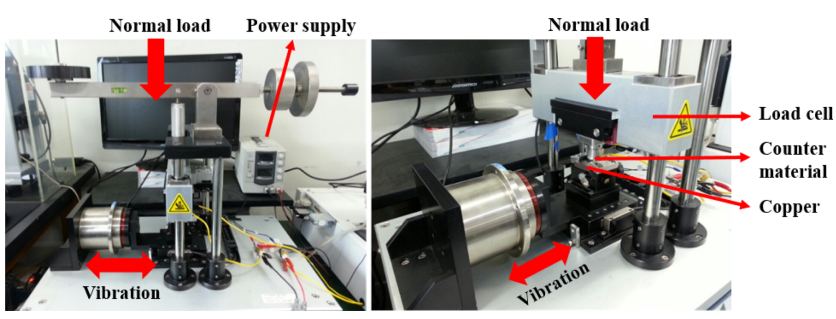

Fig. 2. Experimental set-up for the fretting test. The four-point probe method was employed to measure the electrical contact resistance during the fretting test.

$4 \mathrm{~Hz}$, and displacement amplitude of $300 \mu \mathrm{m}$. During the fretting test, the resistance contact, $\mathrm{R}_{c}$, between the $\mathrm{Cu}$ specimen and S45C steel ball was simultaneously measured using a four-point probe setup. The total wear loss was measured via laser microscopy (VK-8710, Keyence) after the fretting test. Triplicate measurements were conducted, and the obtained wear losses were averaged.

\section{Theoretical calculation}

Fabbro et al. [23] suggested a shock wave pressure model based on an assumption of constant laser power density, $I_{0}$. The laser-induced plasma pressure, $P_{0}$, can be described as Eq. (1).

$$
\mathrm{P}_{0}(\mathrm{GPa})=0.01 \sqrt{\frac{\alpha}{2 \alpha+3}} \cdot \sqrt{Z\left(\frac{\mathrm{g}}{\mathrm{cm}^{2} \mathrm{~s}}\right)} \cdot \sqrt{I_{0}\left(\frac{\mathrm{GW}}{\mathrm{cm}^{2}}\right)}
$$

where the $\alpha$ is the ratio of absorbed laser energy to the thermal energy of the plasma, and it is considered to be 0.11 when water is the confining medium and copper is the specimen [24]. $Z$ is the shock impedance, defined as $2 / Z=1 /$ $Z_{1}+1 / Z_{2}$. Here, $Z_{1}\left(=1.44 \times 10^{6} \mathrm{~g} / \mathrm{cm}^{2} \mathrm{~s}\right)$ is the shock impedance of $\mathrm{Cu}$ and $Z_{2}\left(=0.165 \times 10^{6} \mathrm{~g} / \mathrm{cm}^{2} \mathrm{~s}\right)$ is the shock impedance of water. Therefore, $P_{0}$ can be estimated to be $2.32 \mathrm{GPa}$ for the laser energy density, $I_{0}$, of $5.3 \mathrm{GW} / \mathrm{cm}^{2}$. The depth of residual stress formed by the LSPw/oC process can be determined by calculating the plastic affected depth (PAD) as proposed by Peyre et al. [25, 26]. It can be calculated by Eq. (2)

$$
\mathrm{PAD}=\frac{p_{\max }-\mathrm{HEL}}{2 \mathrm{HEL}}\left(\frac{C_{e l} C_{p l}}{C_{e l}-C_{p l}} \tau\right)
$$

where $P_{\max }$ is the peak plasma pressure, and HEL is the
Hugoniot elastic limit, which is $0.8 \mathrm{GPa}$ for the high-purity $\mathrm{Cu}$ [27]. $C_{\mathrm{el}}$ and $C_{\mathrm{pl}}$ are the elastic and plastic wave speeds of $\mathrm{Cu}$, and are material constants related to the first Lamé constant of the material, $\lambda$, the shear modulus of the material, $G$, and density of material, $\rho$. These are calculated to be 4,760 $\mathrm{m} / \mathrm{s}$ and $3,944 \mathrm{~m} / \mathrm{s}$, respectively at $300 \mathrm{~K}$ [28]. The $\tau$ is the full-width at the half maximum of the shock wave duration, and it can be determined by multiplying a laser pulse duration of 2.5 [17]. As a result, the PAD can be estimated to be 650 $\mu \mathrm{m}$ for the LSP at a laser energy density of $5.3 \mathrm{GW} / \mathrm{cm}^{2}$.

\section{Results and discussion}

Figure 3 shows the cross-sectional microstructures of the BM and LSP $\mathrm{Cu}$ at laser energy densities of 5.3, 10.6, and $18.6 \mathrm{GW} / \mathrm{cm}^{2}$. At the sub-surface, relatively smaller grain sizes were detected for the $\mathrm{LSP} \mathrm{Cu}$ at 5.3 and $10.6 \mathrm{GW} / \mathrm{cm}^{2}$ compared with those of the BM. Moreover, sharp-edged grains were observed for the LSP $\mathrm{Cu}$ at 5.3 and $10.6 \mathrm{GW} / \mathrm{cm}^{2}$ due to the grain refinement induced by LSPw/oC. However, at $18.6 \mathrm{GW} / \mathrm{cm}^{2}$, relatively larger round-edged grains were observed, as compared with those of the BM. This may have been caused by the melting and recrystallization of $\mathrm{Cu}$ due to the high laser energy density, $18.6 \mathrm{GW} / \mathrm{cm}^{2}$, which has been typically observed when the coating on the sample surface has been removed. The coating layer used in the common LSP processes is intended to reduce the damage to the sample surface. In the LSPw/oC process, however, a large amount of thermal energy can also be applied to the subsurface region when the laser power is increased [29, 30].

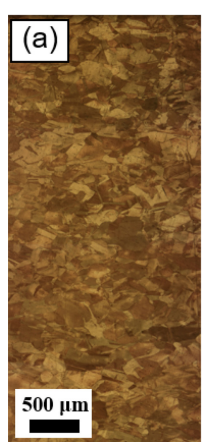

Laser shock-peened surface

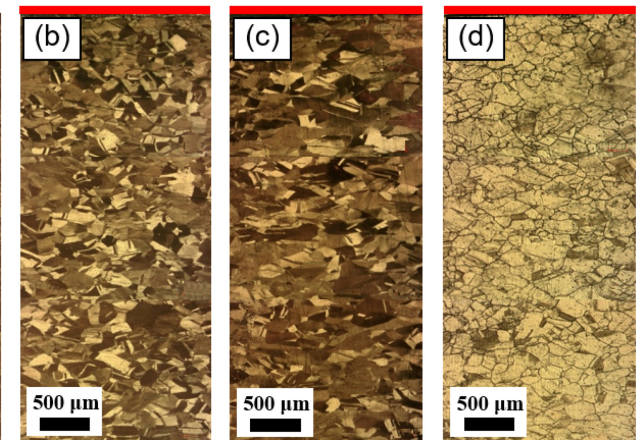

Fig. 3. The cross-sectional microstructures of (a) BM and LSP Cu specimens at the laser energy densities of (b) 5.3, (c) 10.6, and (d) $18.6 \mathrm{GW} / \mathrm{cm}^{2}$. 

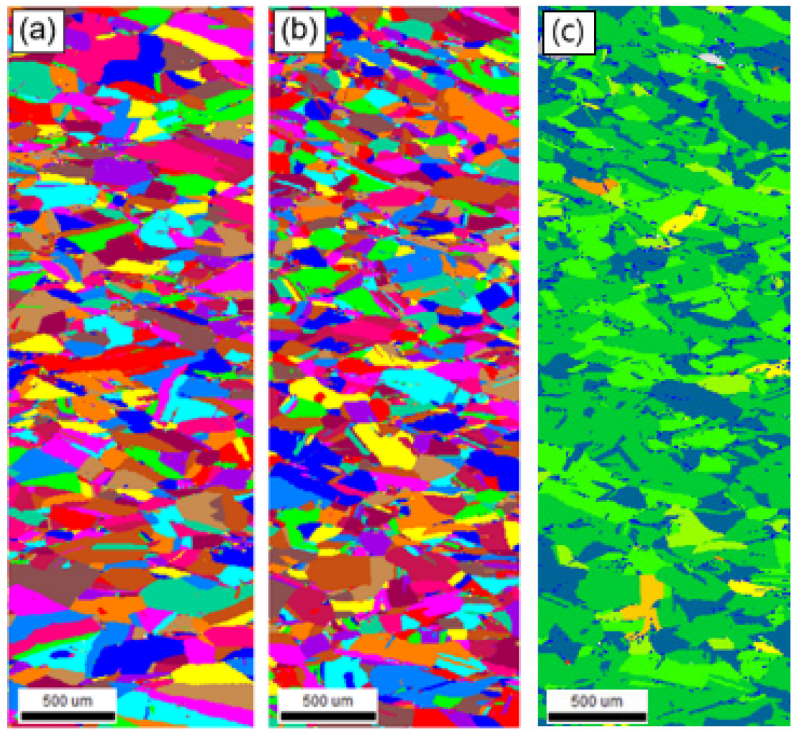

Fig. 4. The EBSD mapping of cross-sectional microstructure of (a) the UCM for BM and (b) the UCM for LSP Cu at $5.3 \mathrm{GW} / \mathrm{cm}^{2}$ (c) the GOS map for LSP $\mathrm{Cu}$ at $5.3 \mathrm{GW} / \mathrm{cm}^{2}$.

Further evidence of recrystallization due to laser thermal energy using $18.6 \mathrm{GW} / \mathrm{cm}^{2}$ was the observation of dislocation density, by TEM analysis [20]. Microstructures exposed to laser energy densities of 5.4 and $10.6 \mathrm{GW} / \mathrm{cm}^{2}$ possess dense dislocation structures, such as dislocation tangles and dislocation walls, which infers significant plastic deformation took place during the LSPw/oC. Those dislocation structures, however, disappeared in the $\mathrm{LSP} / \mathrm{oC}$ sample using $18.6 \mathrm{GW} / \mathrm{cm}^{2}$. Moreover, nano-grains were formed throughout the subsurface region of the sample [20].

Figure 4(a) and (b) show the unique color map (UCM) from the EBSD analysis of the cross-sectional microstructure for $\mathrm{BM}$ and $\mathrm{LSP} \mathrm{Cu}$ at $5.3 \mathrm{GW} / \mathrm{cm}^{2}$. Grain refinement and twin structures were induced by the $\mathrm{LSP} / \mathrm{oC}$ process. At a depth down to $1000 \mu \mathrm{m}$ from the top-surface, the average grain size was found to be $159.3 \mu \mathrm{m}$ for the $\mathrm{BM}$, and it decreased to $99.3 \mu \mathrm{m}$ for the LSP $\mathrm{Cu}$. Grain refinement produced by the LSPw/oC process was detected up to a depth of approximately $1,000 \mu \mathrm{m}$. The average grain size increased to $133.9 \mu \mathrm{m}$ at a depth from 1000 to $1500 \mu \mathrm{m}$ and $159.2 \mu \mathrm{m}$ at a depth from 1,500 to $2,000 \mu \mathrm{m}$. Figure 4(c) displays a grain orientation spread (GOS) map of the LSP $\mathrm{Cu}$ treated at 5.3 $\mathrm{GW} / \mathrm{cm}^{2}$, demonstrating a relatively higher misorientation among grains at the subsurface area where LSP was applied than in the bulk region.

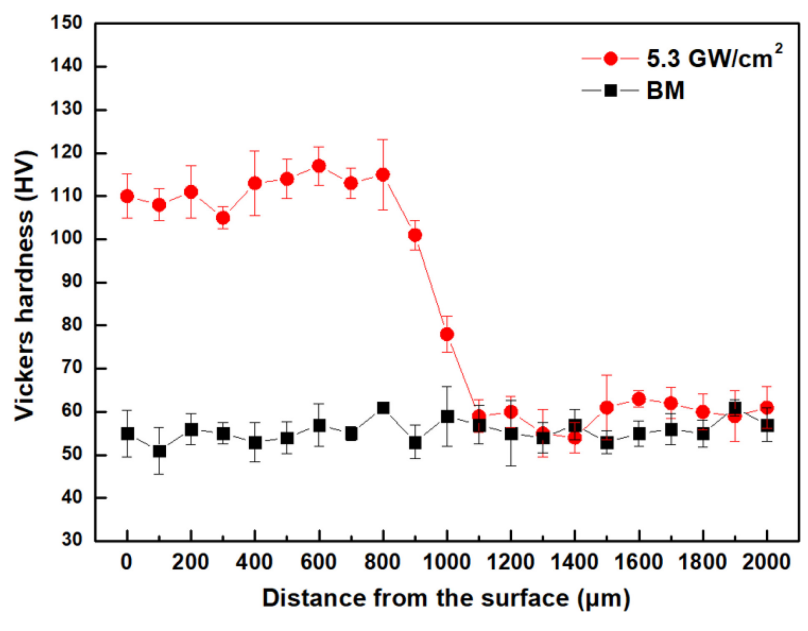

Fig. 5. Microhardness of BM and $\mathrm{LSP} C \mathrm{Cu}$ at $5.3 \mathrm{GW} / \mathrm{cm}^{2}$.

Figure 5 plots the microhardness as a function of distance from the surface, before and after the LSPw/oC process. The surface hardness of the BM was determined to be $55 \mathrm{HV}$, while $110 \mathrm{HV}$ of surface hardness was measured for the LSP $\mathrm{Cu}$ at $5.3 \mathrm{GW} / \mathrm{cm}^{2}$. The increased microhardness was maintained down to a depth of $800 \mu \mathrm{m}$, and it began to decrease thereafter. Eventually, a microhardness comparable to that of the BM was obtained at a depth of $1100 \mu \mathrm{m}$. The increase in microhardness was attributed to the microstructural evolution induced by the LSPw/oC process.

Figure 6 demonstrates the residual stress as a function of depth from the surface for BM and LSP $\mathrm{Cu}$ at the laser energy density of $5.3 \mathrm{GW} / \mathrm{cm}^{2}$. A marginal tensile residual stress was identified at the surface of the BM, while a compressive residual stress, maintained down to a depth of $1,000 \mu \mathrm{m}$, was observed for the LSP Cu. The observations of the microhardness and residual stress coincided well with those in the EBSD analysis in terms of the depth affected by the $\mathrm{LSP}$ w/oC process. The experimental PAD value, which was $650 \mu \mathrm{m}$, was larger than the calculated PAD values based on Fabbro's and Peyre's models. The experimental PAD may have been induced by a combination of thermal and shock wave propagation effects due to the absence of a protective coating layer [31]; this would have resulted in a relatively larger PAD than the calculated PAD, which only considered the shock wave propagation.

The fretting test was conducted for BM and LSP $\mathrm{Cu}$ to estimate wear resistance and the durability of the electrical 


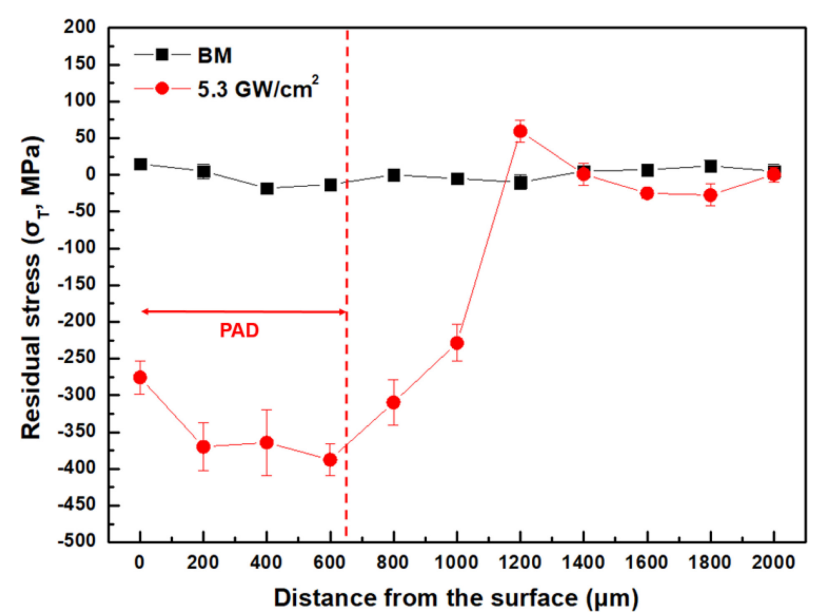

Fig. 6. The residual stress as a function of the depth from the surface for $\mathrm{BM}$ and $\mathrm{LSP} \mathrm{Cu}$ at $5.3 \mathrm{GW} / \mathrm{cm}^{2}$.

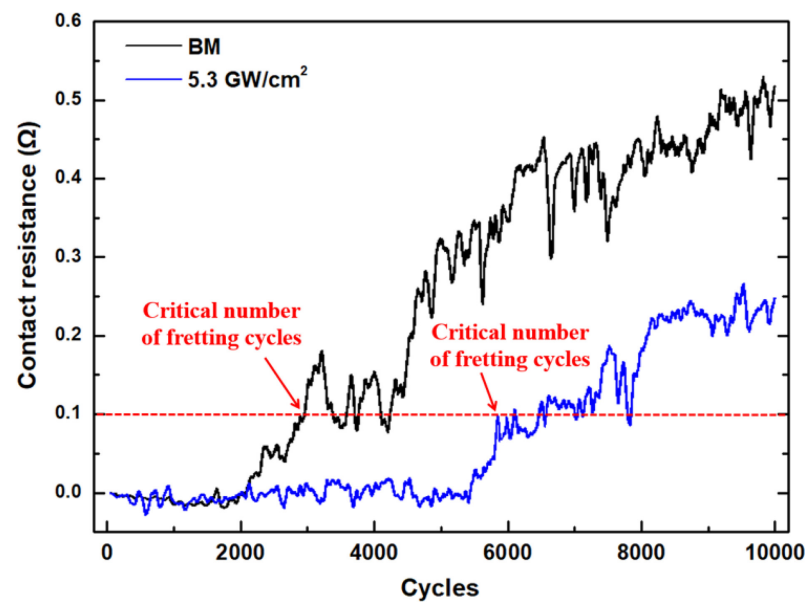

Fig. 7. The contact resistance as a function of the fretting cycles of $\mathrm{BM}$ and $\mathrm{LSP} \mathrm{Cu}$ at $5.3 \mathrm{GW} / \mathrm{cm}^{2}$.

contact. For the BM, the average coefficient of friction (COF) and total wear loss were found to be 0.363 and 1.58 $\times 10^{-3} \mathrm{~mm}^{3}$, respectively. In contrast, for LSP Cu, the average COF was 0.325 , and total wear loss was $0.97 \times 10^{-3} \mathrm{~mm}^{3}$ for $\mathrm{LSP} \mathrm{Cu}$ at $5.3 \mathrm{GW} / \mathrm{cm}^{2}$. Relatively smaller values of $\mathrm{COF}$ and total wear loss were detected in the LSP $\mathrm{Cu}$, compared to those of the BM.

Figure 7 shows the $R_{c}$ as a function of the fretting cycles. In this study, the critical number of fretting cycles is defined as the number of fretting cycles before which an electrical contact failure occurs, and where $\mathrm{R}_{\mathrm{c}}$ exceeds $0.1 \Omega$ for more than 10 subsequent cycles. In the case of BM, the critical number of fretting cycles was 2,930, while the critical number of fretting cycles for LSP $\mathrm{Cu}$ at the laser energy density of $5.3 \mathrm{GW} / \mathrm{cm}^{2}$ was found to be 5,849 . The delay in electrical failure in the LSP $\mathrm{Cu}$ was caused by a smaller amount of wear loss, compared to that of BM. The lower wear loss caused reduced wear debris accumulation and layer generation, which otherwise would have hindered electrical conduction [32]. Therefore, the LSP $\mathrm{Cu}$ maintained a low level of $R_{c}$ showing greater durability.

\section{Conclusion}

In this study, the LSPw/oC process was performed on highpurity copper. At a laser energy density of $5.3 \mathrm{GW} / \mathrm{cm}^{2}$, the laser shock-peened copper showed an increased microhardness and the formation of a compressive residual stress, down to a depth of around $1000 \mu \mathrm{m}$. Additionally, grain refinement and twin structures were observed for the laser shock-peened copper via EBSD pattern analysis. The improvement in mechanical properties and microstructure evolution induced by LSPw/oC in the pure copper resulted in superior wear resistance (i.e., a low coefficient of friction and wear loss) compared to the base metal. This led to low electrical contact resistance, and a delay in the failure of the electrical contact during the fretting test.

\section{Acknowledgement}

This work was supported by INHA UNIVERSITY Research Grant (57011-1)

\section{REFERENCES}

1. D. J. C. M. T. Mannetje, C. U. Murade, D. V. D. Ende, and F. Mugele, Appl. Phys. Lett. 98, 014102 (2011).

2. W. H. Abbott, Proc. 35th Meeting of the IEEE Holm Conf. on Electrical Contacts, p.3, Chicago, IL, USA (1989).

3. Y. Shibata, S. Oohira, S. Masui, S. Sawada, K. Iida, T. Tamai, and Y. Hattori, 26th Int. Conf. on Electrical Contacts (ICEC 2012), p.228, Beijing, China (2012).

4. S. Fouvry, P. Jedrzejczyk, and P. Chalandon, Wear 271, 1524 (2011).

5. M. Braunovic, Ieice T Electron E92.C, 982 (2009).

6. S. Hannel, S. Fouvry, P. Kapsa, and L. Vincent, Wear 249 , 761 (2001). 
7. R. B. Waterhouse, Wear 100, 107 (1984).

8. K. Holmberg and A. Matthews, Coatings tribology : properties, mechanisms, techniques and applications in surface engineering, xv, pp. 560-563, Elsevier Science, Amsterdam ; Boston (2009).

9. R. D. Malucci, Proc. 49th IEEE Holm Conf. on Electrical Contacts, p. 2, Washington, DC, USA (2003).

10. P. L. Y.-S. Shen, J. Gardner, and H. Wiegard, Electrical contact materials: in ASM Handbook Volume 2: Properties and Selection: Nonferrous Alloys and Special-Purpose Materials, $10^{\text {th }}$ ed., pp. 840-868, ASM International, Materials Park, Ohio (1990).

11. W. Ren, P. Wang, J. Song, and G. Zhai, Tribol. Int. 70, 75 (2014).

12. C. Georges, H. Sanchez, N. Semmar, C. BoulmerLeborgne, C. Perrin, and D. Simon, Appl. Surf. Sci. 186, 117 (2002).

13. Å. Kassman Rudolphi and S. Jacobson, Tribol. Int. 30, 165 (1997).

14. M. Antler, Wear 106, 5 (1985).

15. G. T. Flowers, X. Fei, M. J. Bozack, and R. D. Malucci, IEEE T Compon. Pack. T. 27, 65 (2004).

16. A. Senouci, H. Zaidi, J. Frene, A. Bouchoucha, and D. Paulmier, Appl. Surf. Sci. 144-145, 287 (1999).

17. Y. S. Zhang, Z. Han, K. Wang, and K. Lu, Wear 260, 942 (2006).

18. R. Mishra, B. Basu, and R. Balasubramaniam, Mat. Sci. Eng. A-Struct. 373, 370 (2004).

19. A. Krell and D. Klaffke, J. Am. Ceram. Soc. 79, 1139 (1996).

20. C. Park, D. Jung, E.-J. Chun, S. Ahn, H. Jang, and Y.-J.
Kim, Appl. Surf. Sci. 514, 145917 (2020).

21. Y. Cao, Y. C. Shin, and B. Wu, J. Manuf. Sci. Eng. 132, 061010 (2010).

22. A. Umapathi and S. Swaroop, Surf. Coat. Tech. 307, 38 (2016).

23. S. Mall, S. A. Namjoshi, and W. J. Porter, Mat. Sci. Eng AStruct. 383, 334 (2004).

24. H. J. Noh, J. W. Kim, S. M. Lee, and H. Jang, Tribol. Int. 111, 39 (2017).

25. C. Ye, S. Suslov, D. Lin, Y. Liao, X. Fei, and G. J. Cheng, J. Appl. Phys. 110, 083504 (2011).

26. C. S. Montross, T. Wei, L. Ye, G. Clark, and Y.-W. Mai, Int. J. Fatigue 24, 1021 (2002).

27. C. Wang, X. Wang, Y. Xu, and Z. Gao, Int. J. Mech. Sci. 108-109, 104 (2016).

28. C. Ye and G. J. Cheng, J. Manuf. Sci. Eng. 132, 061009 (2010).

29. U. Trdan, J. A. Porro, J. L. Ocaña, and J. Grum, Surf. Coat. Tech. 208, 109 (2012).

30. E. Maawad, Y. Sano, L. Wagner, H. G. Brokmeier, and C. Genzel, Mat. Sci. Eng. A-Struct. 536, 82 (2012).

31. T. H. Kang, K. S. Kim, S. H. Park, and K. A. Lee, Korean J. Met. Mater. 56, 423 (2018).

32. S. Anand Kumar, S. Ganesh Sundara Raman, T. S. N. Sankara Narayanan, and R. Gnanamoorthy, Tribol. Int. 57, 107 (2013).

33. K. Y. Luo, J. Z. Lu, L. F. Zhang, J. W. Zhong, H. B. Guan, and X. M. Qian, Mater. Design 31, 2599 (2010).

34. A. Bag, K. S. Park, and S. H. Choi, Met. Mater. Int. 25, 45 (2018). 